\title{
MODELS FOR ORDINAL HIERARCHICAL CLASSES ANALYSIS
}

\author{
Iwin Leenen, IVen Van Mechelen, and Paul De Boeck \\ KATHOLIEKE UNIVERSITEIT LEUVEN
}

\begin{abstract}
This paper proposes an ordinal generalization of the hierarchical classes model originally proposed by De Boeck and Rosenberg (1998). Any hierarchical classes model implies a decomposition of a twoway two-mode binary array $\mathbf{M}$ into two component matrices, called bundle matrices, which represent the association relation and the set-theoretical relations among the elements of both modes in $\mathbf{M}$. Whereas the original model restricts the bundle matrices to be binary, the ordinal hierarchical classes model assumes that the bundles are ordinal variables with a prespecified number of values. This generalization results in a classification model with classes ordered along ordinal dimensions. The ordinal hierarchical classes model is shown to subsume Coombs and Kao's (1955) model for nonmetric factor analysis. An algorithm is described to fit the model to a given data set and is subsequently evaluated in an extensive simulation study. An application of the model to student housing data is discussed.
\end{abstract}

Key words: hierarchical classes, binary data, scalogram, simulation.

In this paper, a new model for two-way two-mode binary data is presented. The new model is a member of the family of hierarchical classes models and will be called the ordinal hierarchical classes (ORDCLAS) model. Like the original disjunctive hierarchical classes model (De Boeck \& Rosenberg, 1988), its conjunctive variant (Van Mechelen, De Boeck, \& Rosenberg, 1995) and its recently proposed three-way extension (Leenen, Van Mechelen, De Boeck, \& Rosenberg, 1999), an ORDCLAS model represents three types of structural relations in the reconstructed data: the association relation between the modes, an equivalence relation on each mode, yielding a twosided clustering, and a hierarchical (or implication) relation on the clusters at each side.

The new model also is a disjunctive/conjunctive decomposition model, similar to the disjunctive/conjunctive models of Coombs and Kao (1955). The latter models imply a decomposition of a two-way two-mode binary data matrix into a number of biorders or Guttman scales (Doignon, Ducamp, \& Falmagne, 1984; Koppen, 1987 and include a representation of the elements of both modes in a multidimensional space. The ORDCLAS model will be shown to subsume both the (two-way) disjunctive/conjunctive hierarchical classes model and the disjunctive/conjunctive models of Coombs and Kao as special cases. As a consequence, ORDCLAS can be considered a unification of classification models and latent trait models.

This paper is organized in five main sections: Section 1 presents the theory of the ordinal hierarchical classes model. Section 2 describes an algorithm for fitting ORDCLAS models to a data set, and section 3 reports the results of a simulation study to evaluate the algorithm's performance. In section 4 , the new model is illustrated with an application to student housing data. Section 5 discusses the links between ORDCLAS and some related models.

\section{Theory}

In this section, we first consider the common core of the different types of hierarchical classes models (1.1). Next, the ordinal hierarchical classes model is introduced (1.2), and subsequently a graphical representation for the model is presented (1.3). 
TABLE 1.

Hypothetical two-way two-mode binary matrix

\begin{tabular}{lccccccc}
\hline & item $a$ & item $b$ & item $c$ & item $d$ & item $e$ & item $f$ & item $g$ \\
\hline Child 1 & 1 & 0 & 1 & 1 & 1 & 0 & 0 \\
Child 2 & 1 & 1 & 1 & 0 & 1 & 1 & 1 \\
Child 3 & 0 & 0 & 0 & 0 & 1 & 1 & 0 \\
Child 4 & 0 & 0 & 0 & 1 & 1 & 1 & 0 \\
Child 5 & 0 & 0 & 0 & 0 & 0 & 0 & 0 \\
Child 6 & 0 & 0 & 0 & 1 & 1 & 1 & 0 \\
Child 7 & 1 & 0 & 1 & 1 & 1 & 1 & 0 \\
Child 8 & 0 & 0 & 0 & 1 & 1 & 0 & 0 \\
\hline
\end{tabular}

\subsection{Common Core of Hierarchical Classes Models}

Two-way hierarchical classes models are models for two-way two-mode binary data. A hierarchical classes model of a binary $h \times n$ matrix $\mathbf{M}$ includes an $h \times r$ matrix $\mathbf{S}$ and an $n \times r$ matrix $\mathbf{P}$, which by some assocation rule are combined to produce $\mathbf{M}$. The integer $r$ is called the $b$ undle-rank ( $b$-rank) of the model. The $r$ columns of $\mathbf{S}$ (respectively $\mathbf{P}$ ) are called row (resp. column) bundles, and $\mathbf{S}$ (resp. $\mathbf{P}$ ) is called the row (resp. column) bundle matrix. Any hierarchical classes model further requires $\mathbf{S}$ and $\mathbf{P}$ to represent three types of structural relations in $\mathbf{M}$ : association, equivalence, and hierarchy. The hypothetical child by item matrix in Table 1 (with a 1 in cell $i j$ iff child $i$ succeeds in item $j$, and a 0 otherwise) will be used as a guiding example to illustrate these three types of relations.

The association relation is the binary relation between the rows and the columns of $\mathbf{M}$ as defined by the one-entries in $\mathbf{M}$ (i.e., row $i$ is associated with column $j$ iff $m_{i j}=1$ ). In Table 1, for example, Child 1 is associated with item $a$ and not with item $b$.

Two equivalence relations are defined: one on each mode of $\mathbf{M}$. Row $i$ is equivalent with row $i^{\prime}$ in $\mathbf{M}$ iff they are associated with the same set of columns in $\mathbf{M}$. Similarly, column $j$ is equivalent with column $j^{\prime}$ in $\mathbf{M}$ iff they are associated with the same set of rows in $\mathbf{M}$. The resulting equivalence classes are called row (resp. column) classes and imply a partition of the rows (resp. columns). In Table 1, for example, Children 4 and 6 constitute a row (child) class as they solve the same set of items.

Two hierarchical relations are defined separately on the rows and on the columns of $\mathbf{M}$. Row $i$ is hierarchically below row $i^{\prime}$ in $\mathbf{M}$ iff the set of columns associated with $i$ is a proper subset of the set of columns associated with $i^{\prime}$. For example, in Table 1, Child 4 is hierarchically below Child 7 as the former only succeeds in a subset of the items the latter succeeds in. Similarly, column $j$ is hierarchically below column $j^{\prime}$ iff the set of rows associated with $j$ is a proper subset of the set of rows associated with $j^{\prime}$. Item $d$ is below item $e$ as any child that solves $d$ also solves $e$. The relations of equivalence and hierarchy will further be referred to as set-theoretical relations. Row $i$ (resp. column $j$ ) being equivalent or hierarchically below row $i^{\prime}$ (resp. column $j^{\prime}$ ) will be denoted by $i \preceq_{\text {Row }} i^{\prime}$ (resp. $j \preceq_{\text {Col }} j^{\prime}$ ).

\subsection{The Ordinal Hierarchical Classes Model}

In an ordinal hierarchical classes model, the bundles of $\mathbf{S}$ and $\mathbf{P}$ are ordinal variables with (at most) $k$ ( $\geq 2$ ) possible values, $k$ being the position-rank ( $p$-rank) of the model. An ORDCLAS model of b-rank $r$ and p-rank $k$ will be denoted as a $k^{r}$-ORDCLAS model. It is assumed, without loss of generality, that the bundle values range over the integers from 0 to $k-1$.

Two types of ORDCLAS models are considered: a disjunctive and a conjunctive type. Below we will explain how the three types of structural relations are represented by each type. Tables 2 
TABLE 2.

Disjunctive ordinal hierarchical classes model for the matrix in Table 1

\begin{tabular}{ccccccc}
\hline & \multicolumn{3}{c}{ Row Bundles } & & \multicolumn{2}{c}{ Column Bundles } \\
\cline { 2 - 3 } \cline { 5 - 6 } Row Entries & I & II & Column Entries & I & II \\
\hline Child 1 & 2 & 0 & item $a$ & 1 & 1 \\
Child 2 & 0 & 2 & item $b$ & 2 & 1 \\
Child 3 & 0 & 1 & item $c$ & 1 & 1 \\
Child 4 & 1 & 1 & item $d$ & 0 & 2 \\
Child 5 & 0 & 0 & item $e$ & 0 & 0 \\
Child 6 & 1 & 1 & item $f$ & 2 & 0 \\
Child 7 & 2 & 1 & item $g$ & 2 & 1 \\
Child 8 & 1 & 0 & & & \\
\hline
\end{tabular}

and 3 , which present a disjunctive $3^{2}$-ORDCLAS model and a conjunctive $3^{3}$-ORDCLAS model, respectively, for the matrix in Table 1 , are used as a guiding example.

The representation of the association relation in a disjunctive ordinal hierarchical classes model is as follows: Row $i$ is associated with column $j$ iff row $i$ exceeds column $j$ on at least one bundle. Formally, for any row $i$ and column $j$ :

$$
m_{i j}=1 \quad \text { iff } \exists q(1 \leq q \leq r): s_{i q}>p_{j q} .
$$

For example, from the model in Table 2 one can derive that Child 1 succeeds on item $a$ as (s)he exceeds the item on bundle I. Likewise, it can be derived that Child 3 does not succeed on item $d$ as (s)he does not exceed item $d$ on any bundle. In a conjunctive model, row $i$ is associated with column $j$ iff row $i$ exceeds or equals column $j$ on each bundle. Formally, for any row $i$ and column $j$ :

$$
m_{i j}=1 \quad \text { iff } \quad \forall q(1 \leq q \leq r): s_{i q} \geq p_{j q} .
$$

For example, from the model in Table 3, it can be derived that Child 1 succeeds on item $a$ as (s)he exceeds or equals the item on any bundle. Likewise, Child 3 does not succeed on item $d$ as (s)he is below $d$ on bundle II.

The set-theoretical relations of equivalence and hierarchy are represented identically in the disjunctive and conjunctive ORDCLAS models: $\mathbf{S}$ and $\mathbf{P}$ are restricted to be such that for any two rows $\hat{i}, i^{\prime}$ :

$$
i \preceq_{\text {Row }} i^{\prime} \text { iff } \forall q(1 \leq q \leq r): s_{i q} \leq s_{i^{\prime} q}
$$

TABLE 3.

Conjunctive ordinal hierarchical classes model for the matrix in Table 1

\begin{tabular}{ccccccccr}
\hline & \multicolumn{3}{c}{ Row Bundles } & & & \multicolumn{2}{c}{ Column Bundles } \\
\cline { 2 - 6 } Row Entries & I & II & III & Column Entries & I & II & II \\
\hline Child 1 & 1 & 2 & 1 & & item $a$ & 1 & 1 & 1 \\
Child 2 & 2 & 1 & 2 & item $b$ & 2 & 1 & 1 \\
Child 3 & 0 & 0 & 2 & item $c$ & 1 & 1 & 1 \\
Child 4 & 0 & 2 & 2 & item $d$ & 0 & 2 & 1 \\
Child 5 & 0 & 0 & 0 & item $e$ & 0 & 0 & 1 \\
Child 6 & 0 & 2 & 2 & item $f$ & 0 & 0 & 2 \\
Child 7 & 1 & 2 & 2 & item $g$ & 2 & 1 & 1 \\
Child 8 & 0 & 2 & 1 & & & & \\
\hline
\end{tabular}


and for any two columns $j, j^{\prime}$ :

$$
j \preceq_{\mathrm{Col}} j^{\prime} \quad \text { iff } \quad \forall q(1 \leq q \leq r): p_{j q} \geq p_{j^{\prime} q} .
$$

Equation (3) respectively, (4), implies that equivalent rows (resp. columns) have identical values on each of the $r$ bundles. For example, Children 4 and 6, which are equivalent (see Table 1), have the same values on each bundle in both Tables 2 and 3 . Furthermore, (3) implies that the hierarchical relation on the rows is directly represented by $S$, whereas (4) implies that the hierarchical relation on the columns is inversely represented by $\mathbf{P}$. For example, Child 4 , being hierarchically below Child 7, does not exceed Child 7 on any bundle in the disjunctive and in the conjunctive models of Tables 2 and 3; also, item $d$, being hierarchically below item $e$, exceeds or equals item $e$ on each bundle in both models. The rationale for the inverse representation of the item hierarchy can be clarified by interpreting the bundles as requisites: if success on item $d$ implies success on item $e$, the requisites for success on item $e$ can be expected to be weaker than the requisites for success on item $d$.

Note that the association rule (1) of the disjunctive model includes a strict inequality, whereas the association rule (2) of the conjunctive model includes a nonstrict inequality. As a result, the disjunctive and conjunctive $k^{r}$-ORDCLAS models are dual models for given $r$ and $k$ : From rules (1) and (2), it follows that matrices $\mathbf{S}$ and $\mathbf{P}$ represent a disjunctive $k^{r}$-ORDCLAS model for a matrix $\mathbf{M}$ iff $\mathbf{S}^{\mathrm{c}}$ and $\mathbf{P}^{\mathrm{c}}$ represent a conjunctive $k^{r}$-ORDCLAS model for $\mathbf{M}^{\mathrm{c}}$, with $s_{i q}^{\mathrm{c}}=(k-1)-s_{i q}, p_{j q}^{\mathrm{c}}=(k-1)-p_{j q}$ and $m_{i j}^{\mathrm{c}}=1-m_{i j}$ for any $i, j$, and $q$. Duality of disjunctive and conjunctive models is more generally dealt with by Leenen, Van Mechelen, and De Boeck (1999). Incidentally, one may notice that the inequalities in model formulations (1) and (2) can easily be reversed by switching the row and column modes.

From a general proposition (Leenen, Van Mechelen, \& De Boeck, 1999) and a generalization of a proof by De Boeck and Rosenberg (1988) it follows that for any given p-rank $k$ $(\geq 2$ ) and any binary matrix $\mathbf{M}$, a b-rank $r$ exists such that $\mathbf{M}$ can be represented by a (disjunctive/conjunctive) $k^{r}$-ORDCLAS model. However, it is not generally true that for any given $r$ and $\mathbf{M}$, a p-rank $k$ can be found such that a $k^{r}$-ORDCLAS model exists for $\mathbf{M}$ : For example, a $k^{1}$ ORDCLAS for $\mathbf{M}$ exists iff the data can be represented without error by a Guttman scale (Guttman, 1944). It may further be noted that, in general, the existence of a disjunctive $k^{r}$-ORDCLAS model for a matrix $\mathbf{M}$ is not equivalent with the existence of a conjunctive $k^{r}$-ORDCLAS model of the same matrix $\mathbf{M}$ (and vice versa). For example, for the hypothetical data matrix in Table 1 , a disjunctive $3^{2}$-ORDCLAS model exists, whereas a conjunctive $3^{r}$-ORDCLAS model exists only for $r \geq 3$.

\subsection{Graphical Representation}

The disjunctive and conjunctive ORDCLAS models can be given a graphical representation that accounts for the three types of structural relations in the model. The representation is one in an $r$-dimensional space with each bundle corresponding to a dimension and with the positions for the rows and columns being defined by their bundle patterns. As the position-rank $k$ fixes the number of different positions on a single dimension, $k^{r}$ corresponds to the number of different positions in the $r$-dimensional space. The latter clarifies the notation $k^{r}$ for a $k^{r}$-ORDCLAS model.

Figure 1 shows the graphical representation of the disjunctive model in Table 2, where the upper triangle of each position contains the corresponding columns (in this case, items) and the lower triangle the corresponding rows (in this case, children). As a consequence, the equivalence classes are immediately visible in the representation. Concerning the hierarchical relations, row (class) $i$ is hierarchically above row (class) $i^{\prime}$ iff there exists a path of black arrows from $i$ to $i^{\prime}$; likewise, column (class) $j$ is hierarchically above column (class) $j^{\prime}$ iff a path of white arrows exists from $j$ to $j^{\prime}$. For example, in Figure 1 it can be derived that Child 7 is hierarchically above Child 8 and that item $e$ is hierarchically above any other item. With respect to the association relation, if the b-rank equals 2 , it holds that row $i$ is associated with column $j$ iff column $j$ is 


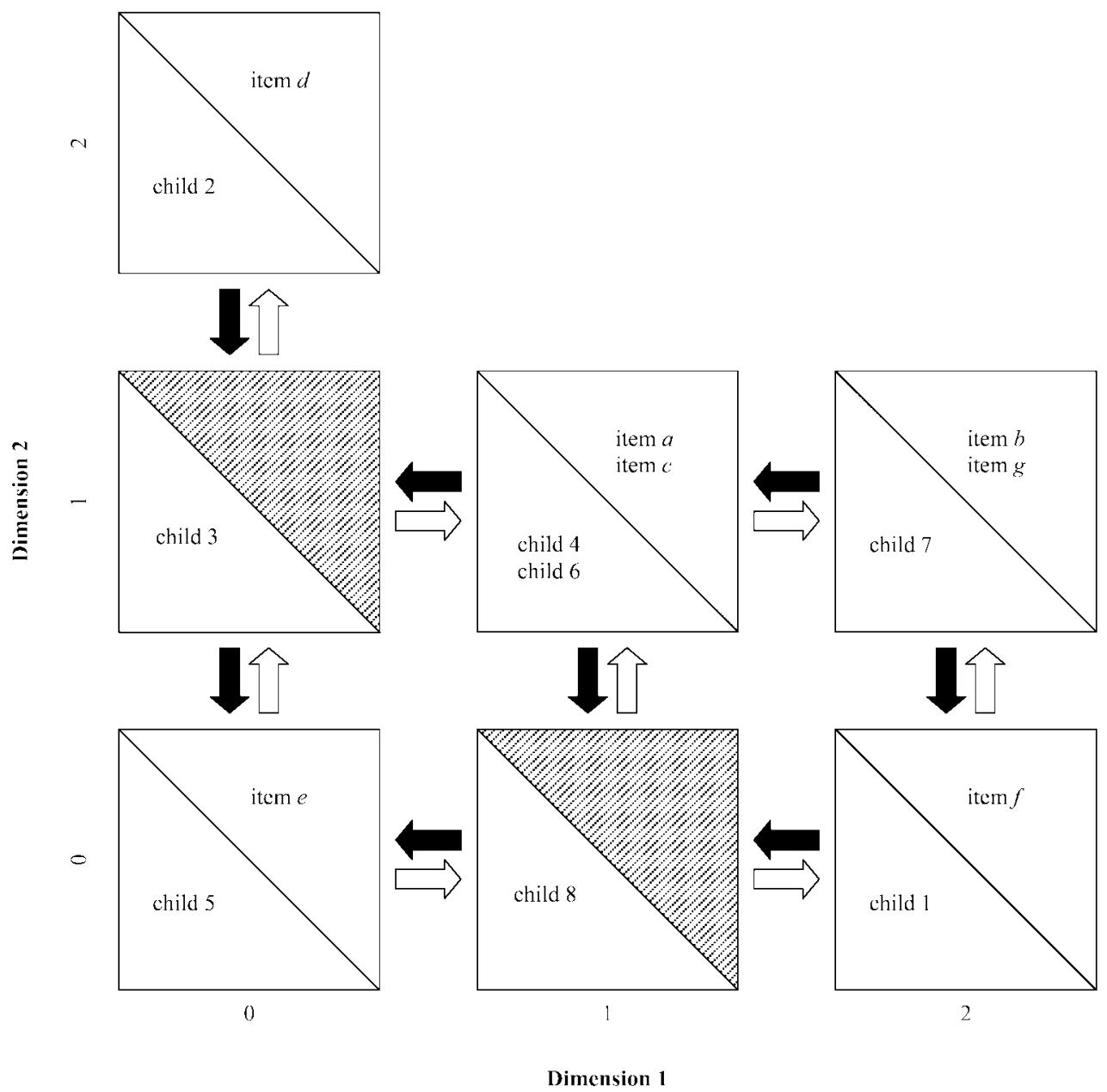

FIGURE 1.

Graphical representation of the disjunctive ORDCLAS model in Table 2. Black and white arrows represent hierarchical relations among rows/children ( $\left.\succ_{\text {Row }}\right)$ and columns/items $\left(\succ_{\mathrm{Col}}\right)$, respectively.

not northeast of row $i$. For example, Children 4 and 6 are associated with items $d, e$ and $f$ but not with items $a, b, c$ and $g$. This idea can be straightforwardly generalized if the bundle-rank exceeds 2.

The graphical representation of the conjunctive $3^{3}$-ORDCLAS model in Table 3 , which would require a three-dimensional space, will not be given here. A graph of a conjunctive model will, however, be presented in the application section. In a conjunctive graph the rows are assigned to the upper triangles and the columns to the lower triangles. The set-theoretical relations are read just as in the disjunctive representation. For the association relation, it holds that row $i$ is associated with column $j$ iff column $j$ is to the southwest of row $i$.

\section{Algorithm}

Given a binary $h \times n$ matrix $\mathbf{D}$ and prespecified values $r$ and $k$ for the b-rank and p-rank, respectively, the ORDCLAS algorithm seeks a binary $h \times n$ matrix $\mathbf{M}$, which can be represented by a conjunctive $k^{r}$-ORDCLAS model, such that the loss function

$$
f(\mathbf{M})=\sum_{i=1}^{h} \sum_{j=1}^{n}\left(d_{i j}-m_{i j}\right)^{2}
$$


is minimized. As $\mathbf{D}$ and $\mathbf{M}$ are binary, (5) can be considered both a least-squares and a leastabsolute deviations loss function (Carroll \& Chaturvedi, 1995). Although the ORDCLAS algorithm primarily fits the conjunctive $k^{r}$-ORDCLAS model, it may be used to fit the disjunctive $k^{r}$-ORDCLAS model as well, because of the duality of disjunctive and conjunctive $k^{r}$-ORDCLAS models.

The algorithm successively executes two main routines: The first one is a minimization routine, which looks for bundle matrices $\mathbf{S}$ and $\mathbf{P}$ that combine by (2) to produce $\mathbf{M}$ for which (5) is minimal. The second routine modifies, if necessary, the matrices $\mathbf{S}$ and $\mathbf{P}$ obtained from the first routine so as to represent the set-theoretical relations in $\mathbf{M}$.

The minimization routine starts from an initial configuration for one of the bundle matrices. This initial configuration can be obtained ( $p s e u d o-$ )randomly or rationally by a built-in heuristic in the algorithm, or can be supplied by the user. In a random initial configuration for the row (resp. column) bundle matrix $\mathbf{S}^{(0)}$ (resp. $\mathbf{P}^{(0)}$ ) the values $s_{i q}^{(0)}$ (resp. $p_{j q}^{(0)}$ ) are independent realizations of a discrete variable uniformly distributed on the set $\{0, \ldots, k-1\}$. (Other discrete distributions can be considered, particularly if some prior knowledge is available.) A rational initial configuration is constructed by using the final solution for the row (resp. column) bundle matrix obtained in a $k^{r-1}$-ORDCLAS analysis to which some new row (resp. column) bundle is added. In particular, the following two steps are executed to obtain an initial row bundle matrix (the procedure for obtaining an initial column bundle matrix is similar): In the first step (a), $n$ candidate row bundles $\mathbf{s}^{(j)}(j=1, \ldots, n)$ are constructed as follows: Regarding bundle $\mathbf{s}^{(j)}$, we start from column $j$ in D, and initialize

$$
s_{i}^{(j)}=\left\{\begin{array}{lll}
0 & \text { iff } & d_{i j}=0 \\
1 & \text { iff } & d_{i j}=1
\end{array}\right.
$$

$\mathbf{s}^{(j)}$ induces an ordered partition of the objects into two subsets $S_{0}$ and $S_{1}$ with $S_{v}=\left\{i \mid d_{i j}=v\right\}$ $(v=0,1)$. For $k>2$, this ordered partition is subsequently refined to an ordered partition into 3 subsets by splitting one of the sets $S_{v}$. In particular, all partitions of $S_{v}$ are considered as induced by all other columns $j^{\prime}$ of $\mathbf{D}$; this procedure yields subsets $S_{v 0}^{j^{\prime}}=S_{v} \cap\left\{i \mid d_{i j^{\prime}}=0\right\}$ and $S_{v 1}^{j^{\prime}}=S_{v} \cap\left\{i \mid d_{i j^{\prime}}=1\right\}$. Eventually, among all possible values of $v$ and $j^{\prime}$, the split is selected for which $\min \left(\# S_{v 0}^{j^{\prime}}, \# S_{v 1}^{j^{\prime}}\right.$ ) (with \# denoting cardinality) is largest. (Ties are broken by retaining the split that was first evaluated.) Assume, without loss of generality, that split $\left(S_{00}^{j^{\prime}}, S_{01}^{j^{\prime}}\right)$ is selected. We then redefine

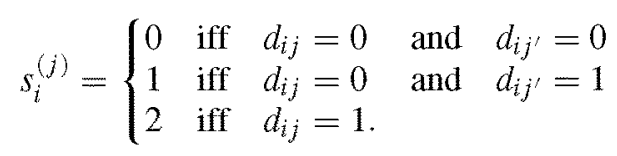

This process of refining the ordered partition is repeated until a partition in $k$ sets is reached, which implies a bundle $\mathbf{s}^{(j)}$ with values on $\{0, \ldots, k-1\}$. As a second step (b) in obtaining an initial row bundle matrix, $n$ candidate row bundle matrices, $s_{j}^{(0)}(j=1, \ldots, n)$, are constructed, where the first $r-1$ bundles of $\mathbf{s}_{j}^{(0)}(j=1, \ldots, n)$ are the bundles of the row bundle matrix of the solution in the previous b-rank $r-1$ (and p-rank $k$ ), and the $r$-th bundle is the new row bundle $\mathbf{s}^{(j)}$ constructed in Step (a). Subsequently, the optimal estimate for the column bundle matrix $\mathbf{P}_{j}^{(0)}$ is found conditionally upon $\mathbf{S}_{j}^{(0)}(j=1, \ldots, n)$ and, eventually, the row bundle matrix $\mathbf{S}_{j}^{(0)}{ }^{j}$ is selected which, combined with $\mathbf{P}_{j}^{(0)}$, yields the matrix $\mathbf{M}$ with minimal value on (5).

The minimization routine proceeds with an alternating least-squares procedure. Assuming, without loss of generality, that an initial configuration, $\mathbf{S}^{(0)}$, has been obtained for the row bundle matrix, the procedure looks, conditionally upon $\mathbf{S}^{(0)}$, for the optimal row bundle matrix $\mathbf{P}^{(0)}$ that minimizes (5). In the next steps, $\mathbf{S}^{(w)}$ is reestimated conditionally upon $\mathbf{P}^{(w-1)}$, and $\mathbf{P}^{(w)}$ is reestimated conditionally upon $\mathbf{S}^{(w)}(w=1,2, \ldots)$. This alternating procedure continues until no further improvement in the loss function (5) is observed. 
With respect to the minimization itself, an analysis of the loss function shows that this function is separable (Chaturvedi \& Carroll, 1994): From the association rule (2), it is clear that

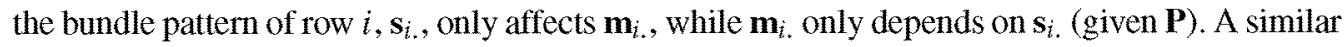
relation holds for the bundle pattern $\mathbf{p}_{j .}$ and $\mathbf{m}_{. j}$. Consequently, an optimal estimate of $\mathbf{S}$ (resp. $\mathbf{P}$ ) conditionally upon $\mathbf{P}$ (resp. S) may be found by a sequential (conditionally optimal) estimation of the bundle patterns $\mathbf{s}_{i .}$. ( $\mathbf{p}_{j}$.) of the $h$ rows (resp. $n$ columns). A conditionally optimal estimate of a bundle pattern is obtained by a branch-and-bound procedure (similar to the one described by Leenen \& Van Mechelen, 1998, for variables having 2 rather than $k$ values). The bounding is based on the number of false negatives, which never decreases when $s_{i q}$ decreases or $p_{j q}$ increases $(i=1, \ldots, h ; j=1, \ldots, n ; q=1, \ldots, r)$. When finding a conditionally optimal row bundle pattern, for example, the latter implies that if the number of false negatives associated with some bundle pattern $\mathbf{s}_{i}$. is known to exceed $f\left(\mathbf{s}_{i .}^{\prime}, \mathbf{P}\right)$, where $\mathbf{s}_{i .}^{\prime}$ is the current best estimate, then bundle patterns $\mathbf{s}_{i .}^{\prime \prime}$ for which $s_{i q}^{\prime \prime} \leq s_{i q}(q=1, \ldots, r)$ need not to be considered.

The second main routine adds the set-theorerical relations to the $\mathbf{S}$ and $\mathbf{P}$ that have been found in the minimization routine. This task is accomplished by minimizing each entry $s_{i q}$ and maximizing each entry $p_{j q}$ (within the range 0 to $k-1$ ) under the restriction that $\mathbf{S}$ and $\mathbf{P}$ do not change the association relation in $\mathbf{M}$. For, if $i \preceq_{\mathrm{Row}} i^{\prime}$ for some $i$ and $i^{\prime}$, whereas a bundle $q$ exists with $s_{i q}>s_{i^{\prime} q}$, then lowering the value of $s_{i q}$ to $s_{i^{\prime} q}$ does not affect $\mathbf{M}$ while $\mathbf{S}$ is changed to represent correctly the set-theoretical relation $i \preceq_{\text {Row }} i^{\prime}$. (An analogous statement holds for the column bundle matrix $\mathbf{P}$.)

\section{Simulation Study}

The aim of the present simulation study is twofold: First, the ORDCLAS algorithm is evaluated with respect to both goodness-of-fit and goodness-of-recovery. Second, a heuristic for model selection is evaluated, to choose a model among several ORDCLAS models of different $b$-ranks and/or p-ranks. In subsection 3.1, the design of the simulation study is outlined. The results are presented in subsection 3.2 (goodness-of-fit), subsection 3.3 (goodness-of-recovery), and subsection 3.4 (rank selection).

\subsection{Design and Procedure}

Three different types of binary $h \times n$ matrices are to be distinguished in this simulation study: a true matrix $\mathbf{T}$, which can be represented by a conjunctive $\kappa^{\rho}$-ORDCLAS model ( $\kappa$ and $\rho$ both being integers); a data matrix $\mathbf{D}$, which equals $\mathbf{T}$ perturbed with error; and the model matrix $\mathrm{M}$ produced by the algorithm, which can be represented by a conjunctive $k^{r}$-ORDCLAS model $(k$ and $r$ both being integers).

Six parameters were systematically varied in a complete six-factorial design:

1. the Size, $h \times n$, of $\mathbf{T}, \mathbf{D}$ and $\mathbf{M}$, at 6 levels: $30 \times 15,50 \times 15,250 \times 15,30 \times 30,50 \times 30$, $250 \times 30$;

2. the True $b$-rank, $\rho$, which is the $\mathbf{b}$-rank of the conjunctive ORDCLAS model for $\mathbf{T}$, at 3 levels: $1,2,3$;

3. the True p-rank, $\kappa$, which is the p-rank of the conjunctive ORDCLAS model for $\mathbf{T}$, at 3 levels: 2, 3, 5;

4. the Error level, $\varepsilon$, which is the proportion of cells $d_{i j}$ differing from $t_{i j}$, at 5 levels: $.00, .01$, $.05, .10, .20$.

5. the Analysis b-rank, $r$, which is the b-rank of the conjunctive ORDCLAS model for $\mathbf{M}$ at 4 levels: 1, 2, 3, 4;

6. the Analysis p-rank, $k$, which is the p-rank of the conjunctive ORDCLAS model for $\mathbf{M}$ at 5 levels: $2,3,4,5,6$. 
Size and Error level are considered random effects, whereas the b-rank and p-rank are considered fixed.

For each combination of Size $h \times n$, True b-rank $\rho$, True p-rank $\kappa$, and Error level $\varepsilon, 20$ pairs of an $h \times \rho$ matrix $\mathrm{S}$ and an $n \times \rho$ matrix $\mathrm{P}$ were generated with

$$
\begin{array}{ll}
S_{i q} \stackrel{\text { i.i.d. }}{\sim} \operatorname{Bin}(\kappa-1, \pi) & (i=1, \ldots, h ; q=1, \ldots, \rho) \\
P_{j q} \stackrel{\text { i.i.d. }}{\sim} \operatorname{Bin}(\kappa-1,1-\pi) & (j=1, \ldots, n ; q=1, \ldots, \rho)
\end{array}
$$

and $\pi$ defined so that $\operatorname{Pr}\left(S_{i q} \geq P_{j q}\right)=\sqrt[p]{.5}$. Bin $(\cdot, \cdot)$ denotes the binomial distribution. Subsequently, matrices $\mathbf{T}$ were calculated by combining $\mathbf{S}$ and $\mathbf{P}$ by the conjunctive association rule (2). Note that by the proposed value for $\pi$, the expected proportion of one-entries in $\mathbf{T}$ equals 5 . Next, a data matrix $\mathbf{D}$ was constructed from each $\mathbf{T}$ by altering the value of a randomly selected set of entries in $\mathbf{T}$, consisting of a proportion $\varepsilon$ of the total number of entries. Finally, the oRDCLAS algorithm (with the rational heuristic for the initial configuration) was applied to find for each matrix D, 4 (analysis b-ranks) $\times 5$ (analysis p-ranks) $=20$ matrices $\mathbf{M}$, which can be represented by conjunctive $k^{r}$-ORDCLAS models $(k=2, \ldots, 6 ; r=1, \ldots, 4)$. As a result of this procedure, $20 \times 6$ (Sizes) $\times 3$ (True b-ranks) $\times 3$ (True p-ranks) $\times 5$ (Error levels) $\times 4$ $($ Analysis b-ranks $) \times 5$ (Analysis p-ranks) $=108,000$ different triplets $(\mathbf{T}, \mathbf{D}, \mathbf{M})$ are obtained.

\subsection{Goodness-of-Fit}

For the evaluation of the algorithm with respect to goodness-of-fit and goodness-ofrecovery, only the 5,400 triplets for which $r=\rho$ and $k=\kappa$ are retained. True b-rank and Analysis b-rank are accordingly abbreviated to $b$-rank, and True p-rank and Analysis p-rank are abbreviated to $p$-rank. For each triplet, the proportion of discrepancies between $\mathbf{D}$ and $\mathbf{M}$, which is a badness-of-fit $(B O F)$ statistic, is used to evaluate the fit of the ORDCLAS solutions:

$$
B o F=\frac{\sum_{i=1}^{h} \sum_{j=1}^{n}\left(d_{i j}-m_{i j}\right)^{2}}{h \times n} .
$$

An analysis of variance with $B o F$ as the dependent variable shows a huge main effect for Error Level, the intraclass correlation $\hat{\rho}_{I}$ (Haggard, 1958, pp. 44-72; Kirk, 1982, pp. 162-163, 387389 ) being as large as .97 . Mean badness-of-fit values across the 1,080 observations within each error level are: $.004, .013, .049, .096$ and .181 for $\varepsilon$ equal to $.00, .01, .05, .10$ and .20 , respectively. These findings imply that the algorithm succeeds in finding models that are about as close to the data as the true model is. Note in this respect that, although the global minimum of $B o F$ for a given data set is unknown, the error level $\varepsilon$ (which indicates how close the true model is to the data) implies an upper bound to the global minimum.

In an analysis of the difference between $B o F$ and $\varepsilon$, Error level still shows up as the most important effect $\left(\hat{\rho}_{I}=.32\right)$. This main effect results from the fact that the higher $\varepsilon$ is, the easier it is for the algorithm to find a model that is closer to the data than the true model is to the data. Furthermore, the effect is qualified by the interactions of b-rank $\times$ Error level $\left(\hat{\rho}_{I}=.19\right)$, Size $\times$ Error level $\left(\hat{\rho}_{I}=.12\right)$ and $\mathrm{p}$-rank $\times$ Error level $\left(\hat{\rho}_{I}=.07\right)$, resulting from the Error level effects being less important for higher b-ranks, matrices with few elements in the smallest mode, and higher p-ranks, respectively: Fitting complex models to small data sets results in overfitting, with the models being considerably closer to the data than is the true model. Other effects are not discussed: In this and the following analyses of variance, only effects accounting for at least $5 \%$ of the variance of the dependent variable are considered (i.e., $\hat{\rho}_{I}$ or $\left.\hat{\omega}^{2} \geq .05\right){ }^{1}$

\footnotetext{
${ }^{1}$ For fixed effects, $\omega^{2}$ is used; for random effects, $\rho_{I}$ is used (Kirk, 1982, pp. 162-163, 387-389). Both statistics can be interpreted as proportions of variance accounted for (Hays, 1994, p. 534).
} 
TABLE 4.

Mean badness-of-recovery at levels of size $\times$ error, b-rank $\times$ error, and $p$-rank $\times$ error

\begin{tabular}{lrrrrrrr}
\hline & & \multicolumn{7}{c}{ Error Level } \\
\cline { 3 - 7 } & & .00 & .01 & .05 & .10 & .20 & Overall \\
\hline Size & $30 \times 15$ & .004 & .005 & .014 & .034 & .106 & .033 \\
& $50 \times 15$ & .005 & .005 & .013 & .029 & .096 & .029 \\
& $250 \times 15$ & .004 & .003 & .010 & .026 & .078 & .024 \\
& $30 \times 30$ & .004 & .005 & .009 & .018 & .071 & .021 \\
& $50 \times 30$ & .005 & .004 & .006 & .013 & .055 & .017 \\
& $250 \times 30$ & .005 & .003 & .004 & .009 & .036 & .011 \\
\hline b-rank & 1 & .004 & .000 & .001 & .004 & .026 & .006 \\
& 2 & .007 & .005 & .009 & .022 & .078 & .024 \\
p-rank & 3 & .007 & .008 & .018 & .038 & .118 & .038 \\
& 2 & .003 & .001 & .002 & .006 & .037 & .010 \\
& 3 & .006 & .006 & .010 & .022 & .080 & .025 \\
\hline Overall & 5 & .004 & .006 & .016 & .035 & .104 & .033 \\
\hline
\end{tabular}

\subsection{Goodness-of-Recovery}

Goodness-of-recovery will be investigated with respect to the association relation, the equivalence relations, and the hierarchical relations.

For each triplet (T, D, M) with $\rho=r$ and $\kappa=k$, the proportion of discrepancies between $\mathbf{T}$ and $\mathbf{M}$ was calculated as a measure of the badness-of-recovery $(B o R)$ of the association relation:

$$
B o R=\frac{\sum_{i=1}^{h} \sum_{j=1}^{n}\left(t_{i j}-m_{i j}\right)^{2}}{h \times n}
$$

The mean $B o R$ across the 5,400 observations equals .023 . An analysis of variance with $B o R$ as the dependent variable reveals important main effects of Error level $\left(\hat{\rho}_{I}=.44\right)$ and $b$-rank $\left(\hat{\omega}^{2}=.06\right)$. Badness-of-recovery clearly increases with higher error levels and higher $b$-ranks (also, see Table 4). These main effects are qualified, though, by b-rank $\times$ Error level $\left(\hat{\rho}_{I}=.17\right)$, p-rank $\times$ Error level $\left(\hat{\rho}_{I}=.10\right)$, and Size $\times$ Error level $\left(\hat{\rho}_{I}=.06\right)$ interactions. The effect of Error level considerably increases the higher the b-rank, the higher the p-rank, and the fewer the number of elements in the smallest mode: Fitting complex models (i.e., models with high b-rank and p-rank) to error-perturbed data sets often yields a poor recovery also if the underlying true model itself is complex. The other effects in the analysis accounted for less than $5 \%$ of the variance of $B O R$.

With respect to the recovery of the equivalence relation, the corrected Rand index (Hubert \& Arabie, 1985) is used to compare the partition of the set of row (resp. column) elements in the ORDCLAS model for the matrix $\mathbf{T}$ with the partition of the set of row (resp. column) elements in the ORDCLAS model for the matrix $\mathbf{M}$. This index equals 1 in case the two partitions are identical and 0 if the two partitions correspond no more than expected by chance. As the results were very similar for the row and the column side, a combined corrected Rand index $(c-C R I)$ was calculated by taking the average corrected Rand index for the row and the column equivalence relation, weighted by the number of rows and columns, respectively.

The mean $c$-CRI equals .824, implying a high correspondence between the true and reconstructed equivalence relations. An analysis of variance with $c$ - $C R I$ as dependent variable shows important main effects of Error level $\left(\hat{\rho}_{I}=.37\right)$, b-rank $\left(\hat{\omega}^{2}=.14\right)$, and p-rank $\left(\hat{\omega}^{2}=.09\right)$, the $c$-CRI decreasing with increasing Error level, b-rank and p-rank, respectively (also, see Table 5). Additionally, important interactions were found: Error level $\times$ b-rank $\left(\hat{\rho}_{I}=.11\right)$ and Error level 
TABLE 5 .

Mean combined corrected Rand index at levels of b-rank $x$ error and p-rank $x$ error

\begin{tabular}{|c|c|c|c|c|c|c|c|}
\hline & & \multicolumn{6}{|c|}{ Error Level } \\
\hline & & .00 & .01 & .05 & .10 & .20 & Overall \\
\hline \multirow[t]{3}{*}{ b-rank } & 1 & 1.000 & .995 & .982 & .955 & .832 & .953 \\
\hline & 2 & .923 & .933 & .878 & .778 & .521 & .806 \\
\hline & 3 & .906 & .892 & .777 & .638 & .349 & .713 \\
\hline \multirow[t]{3}{*}{ p-rank } & 2 & .972 & .985 & .975 & .940 & .793 & .932 \\
\hline & 3 & .925 & .925 & .873 & .785 & .544 & .810 \\
\hline & 5 & .931 & 910 & .794 & .646 & .367 & .730 \\
\hline Overall & & .943 & .940 & .879 & .790 & .568 & .824 \\
\hline
\end{tabular}

$\times$ p-rank $\left(\hat{\rho}_{I}=.10\right)$ which indicate that the decrease rate of $c-C R I$ as a function of Error level is stronger with increasing $b$-rank and p-rank, respectively. The other effects accounted for less than $5 \%$ of the variance in $c-C R I$.

To quantify the goodness-of-recovery of the hierarchical relations, we first define the row hierarchy matrix associated with an $h \times n$ binary matrix $\mathbf{A}$ as the $h \times h$ binary matrix $\mathbf{U}^{(\mathbf{A})}$ with $u_{i i^{\prime}}^{(\mathbf{A})}=1$ iff row $i$ is hierarchically below row $i^{\prime}$ in $\mathbf{A}$; likewise, an entry $v_{j j^{\prime}}^{(\mathbf{A})}$ in the $n \times n$ column hierarchy matrix $\mathbf{V}^{(\mathbf{A})}$ equals 1 iff column $j$ is hierarchically below column $j^{\prime}$ in $\mathbf{A}$. Row hierarchy matrices were constructed for $\mathbf{T}$ and $\mathbf{M}$; subsequently, they were compared to obtain a badness-of-hierarchy-recovery (BoHR) statistic for the rows:

$$
B o H R=\frac{\sum_{i=1}^{h} \sum_{i^{\prime}=1}^{h}\left(u_{i i^{\prime}}^{(\mathbf{T})}-u_{i i^{\prime}}^{(\mathbf{M})}\right)^{2}}{h(h-1)} .
$$

Similarly, a $B o H R$ statistic for the columns was defined, and a weighted average of both statistics (weighted by the number of elements in each mode), denoted $c-B o H R$, was used to evaluate the algorithm's performance.

The mean value on $c-B o H R$ equals .050 , which implies that, in general, the reconstructed hierarchy recovers $95 \%$ of the true hierarchy. An analysis of variance with $c-B o H R$ as a dependent variable reveals important main effects for Error level $\left(\hat{\rho}_{I}=.37\right)$, b-rank $\left(\hat{\omega}^{2}=.14\right)$ and p-rank $\left(\hat{\omega}^{2}=.07\right)$, with the recovery being worse if the error level, $\mathrm{b}$-rank, or p-rank increases. Additionally, there are important Error level $\times \mathrm{b}$-rank $\left(\hat{\rho}_{I}=.13\right)$ and Error level $\times \mathrm{p}$-rank $\left(\hat{\rho}_{I}=.07\right)$ interactions, which results from Error level having a larger effect at higher b-ranks and higher

TABLE 6.

Mean badness-of-hierarchy-recovery at levels of b-rank $\times$ error and p-rank $\times$ error

\begin{tabular}{|c|c|c|c|c|c|c|c|}
\hline & & \multicolumn{6}{|c|}{ Error Level } \\
\hline & & .00 & .01 & .05 & .10 & .20 & Overall \\
\hline \multirow[t]{3}{*}{ b-rank } & 1 & .000 & .001 & .004 & .010 & .038 & .010 \\
\hline & 2 & .028 & .024 & .035 & .064 & .147 & .059 \\
\hline & 3 & .027 & .031 & .057 & .094 & .192 & .080 \\
\hline \multirow[t]{3}{*}{ p-rank } & 2 & .011 & .005 & .009 & .017 & .066 & .022 \\
\hline & 3 & .027 & .027 & .038 & .064 & .141 & .060 \\
\hline & 5 & .018 & .023 & .049 & .086 & .170 & .069 \\
\hline Overall & & .018 & .018 & .032 & .056 & .126 & .050 \\
\hline
\end{tabular}


p-ranks (as shown in Table 6). Any other effects in the analysis accounted for less than $5 \%$ of the variance in $C-B o H R$.

\subsection{Rank Selection}

As with the number of factors in factor analysis and the number of clusters in cluster analysis, in most applications neither the true b-rank nor the true p-rank are ever known. In this section, a formal heuristic for rank selection is presented for cases in which several ORDCLAS models with varying $b$-rank and $p$-rank have been obtained; this heuristic is further evaluated according to the match between the selected model and the true model. The heuristic will be called a pseudo-binomial rule, because it is based on a pseudo-binomial test, similar to the pseudo $F$ tests to select the number of clusters in $k$-means cluster analysis (Hartigan, 1975, pp. 89-91; MacQueen, 1967). This pseudo-binomial test is a generalization of a similar heuristic used for rank selection in HICLAS (Leenen \& Van Mechelen, 2001). For the HICLAS case, this heuristic came up as the best in picking up the true bundle rank and in selecting a model with the highest goodness-of-recovery as compared to a number of other selection heuristics (such as, amongst others, the well-known scree test).

Assume that an $h \times n$ binary matrix $\mathbf{D}$ has been subjected to ORDCLAS -analyses with b-rank varying from 1 to $R$ and p-rank from 2 to $K$, yielding matrices $\mathbf{M}_{r, k}(r=1, \ldots, R$, $k=2, \ldots, K)$. Assume further that for each $\mathbf{M}_{r, k}$ the value of the loss function (5), $f\left(\mathbf{M}_{r, k}\right)$, and the badness-of-fit index defined in $(6), B o F_{r, k}$, have been calculated. The pseudo-binomial rule first selects for each b-rank $r$ the smallest p-rank $k$ for which $f\left(\mathbf{M}_{r, k+1}\right)$ exceeds the first percentile of the binomial distribution $\operatorname{Bin}\left(h n, B \circ F_{r, k}\right)$. Let $k[r]$ denote the selected p-rank for b-rank $r$. Next, the smallest $r$ is selected for which $f\left(\mathbf{M}_{r+1, k[r+1]}\right)$ exceeds the first percentile of the binomial distribution $\operatorname{Bin}\left(h n, B o F_{r, k^{*}}\right)$, where $k^{*}=\max (k[r], k[r+1])$. This number $r$ and the associated $k[r]$ are the b-rank respectively p-rank selected by the pseudo-binomial rule.

In the simulation study, each $\mathbf{D}$ was analyzed with $\mathbf{b}$-ranks varying from 1 to 4 and p-ranks varying from 2 to 6 . The pseudo-binomial rule selects in $50.0 \%$ of the cases both the true b-rank $\rho$ and the true p-rank $\kappa$. The true b-rank in itself is selected in $67.6 \%$ of the cases, and the true p-rank in $58.1 \%$. The selected b-rank and p-rank are more likely to be correct if (a) the number of elements in the smaller mode of $\mathrm{D}$ is large and (b) the true b-rank, true p-rank, and the error level are small. The goodness-of-fit and the goodness-of-recovery of the $k^{r}$-ORDCLAS model selected by the pseudo-binomial rule are very close to the corresponding values for the true $\kappa^{\rho}$-ORDCLAS model, with the overall mean $B o F$-statistics and $B o R$-statistics differing less than .001 and .005 , respectively.

\section{Illustrative Application}

Ordinal hierarchical classes models are suitable for the analysis of data that can be assumed to result from a disjunctive or conjunctive combination of underlying dimensional mechanisms. Relevant possible applications include the analysis of person by task success/failure data (disjunctively modeled with bundles referring to underlying strategies or conjunctively modeled with bundles referring to underlying abilities), student by job like/dislike data (disjunctively modeled with bundles referring to underlying vocational interests), and consumer by brand satisfaction/dissatisfaction data (conjunctively modeled with bundles referring to underlying consumer needs).

In this section we present an analysis of person by choice object select/nonselect data (i.e., pick any/n data, Coombs, 1964, p. 295), conjunctively modeled with bundles referring to underlying choice criteria. The data originate from a study on student housing, previously analyzed by Van Mechelen and Van Damme (1994) with the conjunctive hierarchical classes model (Van Mechelen et al., 1995). 
TABLE 7.

Badness-of-fit values for the models for the student housing data as a function of the p-rank and b-rank

\begin{tabular}{ccccc}
\hline & \multicolumn{4}{c}{ b-rank } \\
\cline { 2 - 5 } & 1 & 2 & 3 & 4 \\
\hline p-rank & & & & \\
2 & .30 & .22 & .18 & .15 \\
3 & .25 & .17 & .13 & .10 \\
4 & .23 & .14 & .11 & .08 \\
5 & .22 & .13 & .10 & .08 \\
\hline
\end{tabular}

In this study, 25 index cards with uniform room descriptions from the Housing Service of the University of Leuven were used. Twenty-six second-year psychology students were asked to select from the set of room descriptions those which they would decide to visit when looking for housing. This task resulted in a binary $25 \times 26$ room by student matrix $\mathbf{D}$ with $\mathbf{D}_{i j}=1$ if room $i$ was selected by student $j$ and 0 otherwise.

Conjunctive ORDCLAS models with bundle-ranks varying from 1 through 4 and positionranks from 2 through 5 were fitted to the matrix $\mathbf{D}$ (using both random and rational initial configurations). Table 7 shows the badness-of-fit statistics for each of the 16 models. Although the pseudo-binomial test recommends a $3^{3}$-ORDCLAS model, for simplicity's sake the $3^{2}$-ORDCLAS model is retained for further discussion in this section. The selected model had $16.9 \%$ discrepancies. The graphical representation of the model is given in Figure 2. As explained in section 1, the (numbers of) rooms (rows) are in the upper triangles, whereas the (numbers of) students (columns) are in the lower triangles. Room $i$ is selected by student $j$ iff student $j$ is to the southwest of room $i$ (i.e., iff room $i$ "dominates" student $j$ on each bundle/dimension).

The two dimensions may be conceived as latent choice criteria implicitly applied by the students and can further be given a substantive interpretation using the characteristics included in the room descriptions. As a result, both latent criteria are found to include elementary comfort as a main requisite, with elementary comfort implying the availability of water in the room, and the availability of warm water and kitchen facilities in the house. The majority of the rooms that lack such elementary facilities were located at the lower end of each dimension. The first dimension could further be interpreted according to the quietness of the environment of the student room: If rooms in the front of houses on busy streets were rated 0 , rooms in the back of houses on busy streets or in the front of houses on moderately quiet streets were rated 1 , and rooms in the back of houses on moderately quiet streets or in houses on quiet streets were rated 2, the Spearman rank correlation between these ratings and the positions on the first dimension is .73 (with the rooms lacking elementary comfort omitted). The second dimension could be interpreted according to the magnitude of the rent, with the Spearman rank correlation with price (across the rooms having elementary comfort) being .76. Students differ in the way they implicitly weight the two latent criteria. For example, the four students at position ( 0 busy; 2 cheap) do not attach great importance to quietness and only care about price, whereas for the four students at position ( 2 quiet; 0 expensive) the opposite holds. The student at the highest position ( 2 quiet; 2 cheap), however, is very demanding as (s)he retains only the single room that is both cheap and quiet.

\section{Relations to Other Models}

The ordinal hierarchical classes model can be shown to be a decomposition model. To clarify this assertion, we reconsider association rule (1) of the disjunctive ORDCLAS model. A component relation $\mathbf{M}^{(q)}$ may be defined for each bundle $q(q=1, \ldots, r)$ as follows:

$$
m_{i j}^{(q)}=1 \quad \text { iff } \quad s_{i q}>p_{j q} .
$$



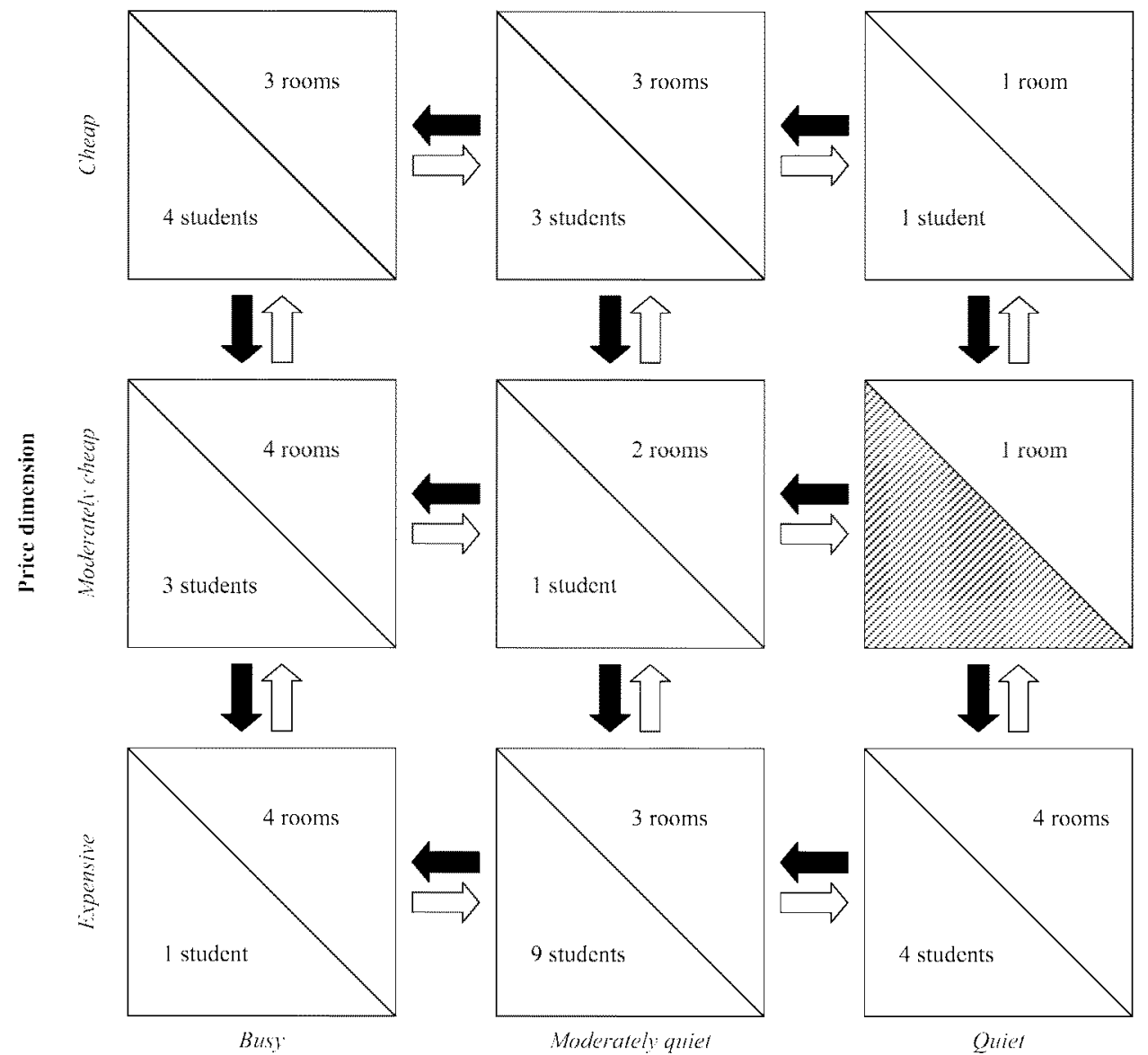

Quietness dimension

FIGURE 2.

Graphical representation of the conjunctive ORDCLAS model for the student housing data. Black and white arrows represent hierarchical relations among rows/rooms ( $>$ Row $)$ and columns/students $\left(\succ_{\mathrm{Col}}\right)$, respectively.

It then holds that $\mathbf{M}$ can be conceived as the disjunctive combination of the component relations $\mathbf{M}^{(q)}$. Similarly, the conjunctive ORDCLAS model may be conceived as a conjunctive combination of component relations (the definition of which slightly differs from (7)). As a disjunctive/conjunctive decomposition model, ORDCLAS is a member of a broad class of disjunctive/conjunctive decomposition models described by Leenen, Van Mechelen, and De Boeck (1999). The generic model discussed there presents a very broad unification of deterministic decomposition models for binary data, including models for nonmetric factor analysis (Coombs \& Kao, 1955), Boolean factor analysis (Mickey, Mundle, \& Engelman, 1983), matching relations (Doignon \& Falmagne, 1984), two-way and three-way hierarchical classes analysis (De Boeck \& Rosenberg, 1988; Van Mechelen et al., 1995; Leenen, Van Mechelen, De Boeck, \& Rosenberg, 1999), and parallelogram analysis (Leenen \& Van Mechelen, 2000), along some very general principles. The paper discusses some general theorems and tools concerning existence, duality of disjunctive/conjunctive models, minimal b-rank, and the determination of exact rather than approximate decompositions, which can be instantiated for ordinal hierarchical classes models as well.

Furthermore, ORDCLAS is closely related to a number of other members of the class of disjunctive/conjunctive decomposition models: It reduces to the original hierarchical classes model 
if $k=2$. In particular, the conjunctive oRDCLAs model with $k=2$ is identical to the model proposed by Van Mechelen et al. (1995); furthermore, though the disjunctive ORDCLAS model with $k=2$ is not identical to the original disjunctive model as proposed by De Boeck and Rosenberg (1988), it is identical to a mathematically equivalent variant of it outlined by Van Mechelen et al. (1995).

For $k \geq \min (h, n)+1,(1)$, respectively (2), is equivalent to the composition axiom of the disjunctive (resp. conjunctive) model of nonmetric factor analysis (Coombs, 1964, p. 252; Coombs \& Kao, 1955) with each of the bundles corresponding to a dimension. Hence, ordinal hierarchical classes models with $k \geq \min (h, n)+1$ can be considered Coombs and Kao models with the additional requirement of the representation of the set-theoretical relations. The latter constitute a unique feature of the ORDCLAS model. The set-theoretical relations may reveal implication relations among elements of the same mode as, for example, extensively used in knowledge space theory (Falmagne, Koppen, Vilano, Doignon, \& Johannesen, 1990).

If $k<\min (h, n)+1$, ORDCLAS models can still be considered set-theoretical Coombs and Kao models, with a restriction, though, on the number of different positions on each dimension. As such, the ORDCLAS model can be considered a bridge between Coombs and Kao's (1955) dimensional model and the HICLAS classification model (De Boeck \& Rosenberg, 1988, Van Mechelen et al., 1995). The lower the p-rank, the closer ORDCLAS comes to a classification model; the higher the p-rank, the closer the model comes to a dimensional model. Intermediate values result in models that can be considered a combination of dimensional and classification models, yielding a number of different classes ordered along a number of dimensions.

Finally, ORDCLAS as a deterministic model is closely related to a number of probabilistic models. As such, the probability matrix decomposition (PMD) models presented by Maris, De Boeck, and Van Mechelen (1996) are probabilistic variants of the original hierarchical classes model (De Boeck \& Rosenberg, 1988; Van Mechelen et al., 1995). Also, several probabilistic extensions of the models of nonmetric factor analysis (Coombs \& Kao, 1955) have been proposed (Maris, 1995, van Leeuwe \& Roskam, 1991). As to the latter, it is interesting to note, from an ORDCLAS point of view, that the estimation of these models may imply a discretization of the ability dimensions into a prespecified number of "nodes" (van Leeuwe \& Roskam, 1991).

\section{References}

Carroll, J.D., \& Chaturvedi, A. (1995). A general approach to clustering and multidimensional scaling of two-way, threeway, or higher-way data. In R.D. Luce, M.D'Zmura, D. Hoffman, G.J. Iverson \& A.K. Romney (Eds.), Geometric representations of perceptual phenomena (pp. 295-318). Mahwah, $\mathrm{NJ}$ : Erlbaum.

Chaturvedi, A., \& Carroll, J.D. (1994). An alternating combinatorial optimization approach to fitting the INDCLUS and generalized INDCLUS models. Joumal of Classification, 11, 155-170.

Coombs, C.H. (1964). A theory of data. New York, NY: Wiley.

Coombs, C.H., \& Kao, R.C. (1955). Nonmetric factor analysis (Engineering Research Bulletin No. 38). Ann Arbor, MI: University of Michigan Press.

De Boeek, P. \& Rosenberg, S. (1988). Hierarchical classes: Model and data analysis. Psychometrika, 53, 361-381.

Doignon, J.-P, \& Falmagne, J.C. (1984). Matching relations and the dimensional structure of social choices. Mathematical Social Sciences, 7, 211-229.

Doignon, J.-P., Ducamp, A., \& Falmagne, J-C. (1984). On realizable biorders and the biorder dimension of a relation. Joumal of Mathematical Psychology, 28, 73-109.

Falmagne, J.C., Koppen, M., Vilano, M., Doignon, J.-P., \& Johannesen, L. (1990). Introduction to knowledge spaces: How to build, test and search them. Psychological Review, 97, 201-224.

Guttman, L. (1944). A basis for scaling qualitative data. Americal Sociological Review, 9, 139-150.

Haggard, E.A. (1958). Intraclass correlation and the analysis of variance. New York, NY: Dryden.

Hartigan, J.A. (1975). Clustering algorithms. New York, NY: Wiley.

Hays, W.L. (1994). Statistics (5th ed.). New York, NY: Harcourt Brace.

Hubert, L., \& Arabie, P. (1985). Comparing partitions. Joumal of Classification, 2, 193-218.

Kirk, R.E. (1982). Experimental design: Procedures for the behavioral sciences (2nd ed.). Belmont, CA: Brooks/Cole.

Koppen, M.G.M. (1987). On finding the bidimension of a relation. Journal of Mathematical Psychology, 31, $155-178$.

Leenen, I., \& Van Mechelen, I. (1998). A branch-and-bound algorithm for Boolean regression. In I. Balderjahn, R. Mathar \& M. Schader (Eds.), Data highways and information flooding, a challenge for classification and data analysis (pp. 164-171). Berlin, Germany: Springer-Verlag.

Leenen, I., \& Van Mechelen, I. (2000). A conjunctive parallelogram model for pick any/n data. Submitted for publication. 
Leenen, I., \& Van Mechelen, I. (2001). An evaluation of two algorithms for hierarchical classes analysis. Journal of Classification, 18, 57-80.

Leenen, I., Van Mechelen, I., \& De Boeck, P. (1999). A generic disjunctive/conjunctive decomposition model for $n$-ary relations. Joumal of Mathematical Psychology, 43, 102-122.

Leenen, I., Van Mechelen, I., De Boeck, P., \& Rosenberg, S. (1999). INDCLAS: A three-way hierarchical classes model. Psychometrika, 64, 9-24.

MacQueen, J. (1967). Some methods for classification and analysis of multivariate observations. In L.M. Le Cam \& J. Neyman (Eds.), Proceedings of the Fifth Berkeley Symposium on Mathematical Statistics and Probability (Vol. 1 , pp. 281-297). Berkeley: University of Califormia Press.

Maris, E. (1995). Psychometric latent response models. Psychometrika, 60, 523-547.

Maris, E., De Boeck, P., \& Van Mechelen, I. (1996). Probability matrix decomposition models. Psychometrika, 61, 7-29.

Mickey, M.R., Mundle, P., \& Engelman, L. (1983). Boolean factor analysis. In W.J. Dixon (Ed.), BMDP statistical software (pp. 538-545, 692). Berkeley: University of California Press.

van Leeuwe, J.F.J., \& Roskam, E.E. (1991). The conjunctive item response model: A probabilistic extension of the Coombs and Kao model. Methodika, 5, 14-32.

Van Mechelen, I., \& Van Damme, G. (1994). A latent criteria model for choice data. Acta Psychologica, 87, 85-94.

Van Mechelen, I., De Boeck, P., \& Rosenberg, S. (1995). The conjunctive model of hierarchical classes. Psychometrika, $60,505-521$.

Manuscript received 26 OCT 1998

Final version received 8 AUG 2000 